\title{
Toric vector bundles: GAGA and Hodge theory
}

\author{
Jonas Stelzig ${ }^{1}$
}

Received: 3 June 2019 / Accepted: 1 September 2020 / Published online: 10 October 2020

(c) The Author(s) 2020

\begin{abstract}
We prove a GAGA-style result for toric vector bundles with smooth base and give an algebraic construction of the Frölicher approximating vector bundle that has recently been introduced by Dan Popovici using analytic techniques. Both results make use of the Rees-bundle construction.
\end{abstract}

\section{Introduction}

A toric variety over a field $k$ is an algebraic variety $X$ over $k$ with a $\mathbb{G}_{m}^{n}$-action that has a dense open orbit on which the group acts simply transitively. A vector bundle on such $X$ is called toric if it is equipped with a $\mathbb{G}_{m}^{n}$-action s.t. the projection is an equivariant map.

Toric varieties and vector bundles are an important source of examples in algebraic geometry. Just as normal toric varieties can be studied by combinatorial data, toric vector bundles (and also more general classes of equivariant sheaves) on a given normal toric variety $X$ have been classified in terms of linear-algebra-data (roughly as vector spaces and filtrations with certain compatibility conditions), c.f. $[10,11,13,14,18]$.

If $k=\mathbb{C}$, for every toric variety $X$ over $\mathbb{C}$ one also has a natural notion of holomorphic toric vector bundles over $X^{\text {an }}$, the latter meaning (the set of complex points of) $X$ seen as a complex analytic space. One obtains an analytification functor:

toric vector bundles on $X \longrightarrow$ holomorphic toric vector bundles on $X^{\text {an }}$

The first main result of this article is that for smooth toric varieties, this functor is an equivalence of categories:

Theorem A For a smooth toric variety $X$ over $\mathbb{C}$, analytification induces an equivalence of categories between algebraic toric vector bundles on $X$ and holomorphic toric vector bundles on $X^{\mathrm{an}}$. The same is true for toric vector bundles with equivariant connections.

Of course, there is the known GAGA-principle by Serre [22], asserting an equivalence of the categories of coherent sheaves on a complex projective variety and its analytification.

Jonas Stelzig

Jonas.Stelzig@math.lmu.de

1 LMU, Munich, Germany 
The above theorem is not a formal consequence of this. For instance, $X$ is not assumed to be projective and even for projective $X$, the equivariant structure is sheaf-theoretically described by an isomorphism of sheaves over $\mathbb{G}_{m}^{n} \times X$, which is not projective.

We do not need the full classification of toric vector bundles. In fact, it is enough for our purposes to consider algebraic toric vector bundles on affine spaces and we include a brief but largely self-contained treatment of these in Sect. 2. A key notion is the Rees-bundle construction, which associates to (suitable) multifiltered vector spaces $\left(V, F_{1}, \ldots, F_{n}\right)$ a toric vector bundle $\xi_{\mathbb{A}^{n}}\left(V, F_{1}, \ldots, F_{n}\right)$ on $\mathbb{A}^{n}$.

Toric vector bundles have also been studied in connection with Hodge theory, see e.g. $[5-7,12,16,17,23,24]$, in part apparently independent of the classification. A basic idea is that, since a Hodge structure is a multifiltered vector space, one may apply Rees-bundle like constructions to it and obtain a toric vector bundle.

Recently in [19], Popovici introduced the so-called Frölicher approximating bundle, in order to give a more conceptual proof of his earlier result that deformation limits of Moishezon manifolds are again Moishezon. This construction associates to every compact complex manifold $X$ and every integer $k$ a holomorphic vector bundle $\mathcal{A}^{k}$ on $\mathbb{C}$ (thus necessarily trivial) which interpolates between the de-Rham cohomology and the degenerating page of the Frölicher spectral sequence. I.e., it comes equipped with distinguished isomorphisms $\mathcal{A}^{k}(h) \cong H_{d R}^{k}(X, \mathbb{C})$ for $h \neq 0$ and $\mathcal{A}^{k}(0) \cong \bigoplus_{p+q=k} E_{\infty}^{p, q}$. The construction is analytic, in particular it involves choosing a metric and introducing Laplace-type pseudo-differential operators for the higher pages of the Frölicher spectral sequence. We show here that this bundle can be seen as a special case of the Rees-bundle construction, thereby giving a purely algebraic description, which makes the equivariant, metric-independent and functorial features of this construction transparent.

Theorem B For a compact complex manifold $X$ and the $k$-th Frölicher approximating bundle $\mathcal{A}^{k}$, there is a canonical isomorphism

$$
\mathcal{A}^{k} \cong \xi_{\mathbb{A}^{1}}\left(H_{d R}^{k}(X, \mathbb{C}), F\right)^{\text {an }},
$$

where $F$ denotes the Hodge-filtration.

\section{Preliminaries}

This section collects some basic notions and result from more general theory which are needed later on. No claim to originality is made here: All results (except possibly for mistakes on the author's behalf) are contained in one or more of [5-7,10-14,16-18,23,24]. If we give only one reference for an omitted proof we do not mean to imply any kind of priority.

\subsection{Definitions}

Throughout all the section, we fix an algebraically closed field $k$ of characteristic zero and denote by $G$ an algebraic group over $k$, which will soon be set to be the algebraic $n$-torus $\mathbb{G}_{m}^{n}$. We first repeat some standard definitions. Even though everything is formulated for algebraic varieties, the reader may note that, mutatis mutandis, most of the definitions make sense in the holomorphic, smooth or even continuous setting. Sections 2 and 3 of [15] may be a helpful reference. 
Definition 1 A $G$-variety is an algebraic variety $X$ over $k$ together with an action $\rho_{X}$ : $G \times X \longrightarrow X$ of the group $G$. If $G=\mathbb{G}_{m}^{n}$ and there is a dense open orbit on which it acts simply transitively, $\left(X, \rho_{X}\right)$ is called a toric variety.

Example 2 Any variety is a $G$-variety for the trivial action. The varieties $\mathbb{G}_{m}^{l} \times \mathbb{A}^{n-l}$ for the natural action of $\mathbb{G}_{m}^{n}$ by multiplication are toric.

Definition 3 An equivariant sheaf (of $\mathcal{O}_{X}$-modules) on a $G$-variety $\left(X, \rho_{X}\right)$ is tuple $(\mathcal{V}, \Phi)$, where $\mathcal{V}$ is a sheaf and $\Phi$ is an isomorphism

$$
\Phi: \rho_{X}^{*} \mathcal{V} \longrightarrow \operatorname{pr}_{2}^{*} \mathcal{V}
$$

of $\mathcal{O}_{G \times X}$-modules that satisfies the cocycle condition

$$
\operatorname{pr}_{23}^{*} \Phi \circ\left(\operatorname{Id}_{G} \times \rho_{X}\right)^{*} \Phi=\left(\mu_{G} \times \operatorname{Id}_{X}\right)^{*} \Phi,
$$

where $\operatorname{pr}_{23}, \operatorname{Id}_{G} \times \rho_{X}, \mu_{G} \times \operatorname{Id}_{X}$ are maps $G \times G \times X \longrightarrow G \times X$ given by projection, action and multiplication respectively.

One may check that if $\mathcal{V}$ is a coherent and locally free, i.e. the sections of a vector bundle $E \longrightarrow X$, this definition is equivalent to requiring $E$ to be a $G$-variety s.t. the action commutes with the projection.

Example 4 Let $\Omega_{X}$ be the sheaf of Kähler differentials. Recall that for any product $X \times Y$ there is a canonical identification $\Omega_{X \times Y} \cong \operatorname{pr}_{X}^{*} \Omega_{X} \oplus \operatorname{pr}_{Y}^{*} \Omega_{Y}$. As a consequence one may equip $\Omega_{X}$ with the structure of an equivariant sheaf via

$$
\Phi_{\Omega}: \rho_{X}^{*} \Omega_{X} \longrightarrow \Omega_{G \times X} \cong \operatorname{pr}_{G}^{*} \Omega_{G} \oplus \operatorname{pr}_{X}^{*} \Omega_{X} \longrightarrow \operatorname{pr}_{X}^{*} \Omega_{X},
$$

where the first map is pullback via $\rho_{X}$ and the second map is projection.

Recall that for a connection $\nabla: \mathcal{V} \longrightarrow \mathcal{V} \otimes \Omega_{X}$ and a map $f: Y \longrightarrow X$, there is a well-defined notion of pullback $f^{*} \nabla: f^{*} \mathcal{V} \longrightarrow f^{*} \mathcal{V} \otimes \Omega_{Y}$ which is again a connection: If locally $\nabla=d+\omega$ with $\omega$ a section of $\Omega_{X}^{1}(\operatorname{End}(\mathcal{V}))$, then $f^{*} \nabla=d+f^{*} \omega$. More globally, one considers $f^{-1} \nabla$ and prolongs it to $f^{*} \mathcal{V}=f^{-1} \mathcal{V} \otimes_{f^{-1}} \mathcal{O}_{X} \mathcal{O}_{Y}$ via the Leibniz-rule.

Definition 5 An equivariant connection on an equivariant sheaf $(\mathcal{V}, \Phi)$ on a toric variety $\left(X, \rho_{X}\right)$ is a connection $\nabla: \mathcal{V} \longrightarrow \mathcal{V} \otimes \Omega_{X}$ such that the following diagram commutes:

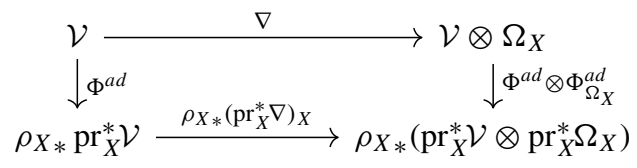

Here, $\left(\operatorname{pr}_{X}^{*} \nabla\right)_{X}$ denotes the composite of $\operatorname{pr}_{X}^{*} \nabla: \operatorname{pr}_{X}^{*} \mathcal{V} \longrightarrow \operatorname{pr}_{X}^{*} \mathcal{V} \otimes \Omega_{G \times X}$ with Id $\otimes$ res where res : $\Omega_{G \times X} \longrightarrow \operatorname{pr}_{X}^{*} \Omega_{X}$ is projection.

On affine schemes, the above notions can, as usual, be translated into commutative algebra and it is this description that will be used later on in this section.

Proposition 6 The global sections functors yield equivalences of categories between: 
1. The category of quasi-coherent equivariant sheaves on an affine toric variety $X=\operatorname{Spec} A$ and the category of $n$-graded A-modules.

2. The category of equivariant vector bundles with connection on a toric variety $X=\operatorname{Spec} A$ and the category of $n$-graded $A$ modules $M$ which are locally free of finite rank and equipped with a map

$$
\nabla: M \longrightarrow M \otimes \Omega_{A},
$$

that satisfies the Leibniz rule and respects the grading. Here, $\Omega_{A}$ denotes the module of Kähler differentials, the generators of which satisfy $\operatorname{deg}\left(d X_{i}\right)=\operatorname{deg}\left(X_{i}\right)$, and the right hand side has the tensor product grading.

The proof is standard and we omit it. ${ }^{1}$ Let us just describe how the $n$-grading on $A$ is defined: The action $\rho_{X}$ induces a coaction $\rho_{X}^{*}: A \longrightarrow A \otimes k\left[z_{1}^{ \pm 1}, \ldots, z_{n}^{ \pm 1}\right]$ and for any multiindex $p=\left(p_{1}, \ldots, p_{n}\right) \in \mathbb{Z}^{n}$, one sets

$$
A^{p}:=\left\{a \in A \mid \rho^{*}(a)=a \otimes z_{q}^{p_{1}} \cdot \ldots \cdot z_{n}^{p_{n}}\right\} .
$$

\subsection{Toric vector bundles on affine and projective spaces}

It is well-known that one can further simplify the commutative algebra from the previous section and describe equivariant sheaves on normal toric varieties in terms of linear algebra. We keep notations from the previous subsection, and recall how this works for the case $X=\mathbb{A}^{n}=\operatorname{Spec} k\left[z_{1}, \ldots, z_{n}\right]$. We will only use these results for $k=\mathbb{C}$, but everything works for algebraically closed fields of characteristic zero.

Notational conventions: We fix some natural number $n$ and denote by for any toric variety $X$ by $\operatorname{Bun}\left(X, \mathbb{G}_{m}^{n}\right)$ the category of locally free coherent equivariant sheaves on $X$ with morphisms equivariant morphisms of sheaves (not necessarily of constant rank). By Fil $k$, we denote the category of finite dimensional $k$-vector spaces $\left(V, F_{1}, \ldots, F_{n}\right)$ with $n$ separated and exhaustive descending filtrations (i.e. $F_{i}^{P}=\{0\}$ and $F_{i}^{p}=V$ for $P \gg 0 \gg p$ ). We sometimes write simply $V$ instead of $\left(V, F_{1}, \ldots, F_{n}\right)$ for an object in Fil ${ }_{k}^{n}$. Morphisms in Fil $_{k}^{n}$ are linear maps $\varphi: V \rightarrow V^{\prime}$ such that $\varphi\left(F_{i}^{p}\right) \subseteq F_{i}^{p}$. Given two filtered vector spaces $(V, F),\left(V^{\prime}, F^{\prime}\right)$, the tensor product $V \otimes_{k} V^{\prime}$ is equipped with the filtration $\left(F \otimes F^{\prime}\right)^{\bullet}=$ $\sum_{p+q=.} F^{p} \otimes_{k} F^{\prime q}$ and this induces a tensor product on $\mathrm{Fil}_{k}^{n}$.

We write $A:=k\left[z_{1}, \ldots, z_{n}\right]$ and $B:=k\left[z_{1}^{ \pm 1}, \ldots, z_{n}^{ \pm 1}\right]$. We view these as equipped with the standard $n$-grading, i.e., $z_{i}^{p}$ has $i$-th degree $p$ and zero else. For a multiindex $p=$ $\left(p_{1}, \ldots, p_{n}\right) \in \mathbb{Z}^{n}$, we write $|p|:=\sum_{i=1}^{n} p_{i}$ and $z^{p}:=z_{1}^{p_{1}} \cdot \ldots \cdot z_{n}^{p_{n}} \in B$. For $n$ filtrations $F_{i}$ on some vector space, we set $F^{p}:=F_{1}^{p_{1}} \cap \ldots \cap F_{n}^{p_{n}}$. Given some $\lambda \in \mathbb{Z}$, we use the notation $\lambda p:=\left(\lambda p_{1}, \ldots, \lambda p_{n}\right)$ and $p \pm_{i} \lambda:=\left(p_{1}, \ldots, p_{i-1}, p_{i} \pm \lambda, p_{i+1}, \ldots, p_{n}\right) \in \mathbb{Z}^{n}$ and similarly with $\geq_{i}$ instead of $\pm_{i}$. We abbreviate $(0, \ldots, 0)$ to just 0 . Also, for $p, r \in \mathbb{Z}^{n}$, we write $p \geq r: \Leftrightarrow p_{i} \geq r_{i} \forall i \in\{1, \ldots, n\}$.

From filtrations to sheaves: Starting from a multi-filtered vector space $\left(V, F_{1}, \ldots, F_{n}\right)$, denote by

$$
\operatorname{Rs}^{n}(V):=F^{0}\left(V \otimes_{k} B\right)=\sum_{p \in \mathbb{Z}^{n}} F^{p} V \otimes_{k} z^{-p} A \subseteq V \otimes_{k} B
$$

1 See e.g. [4, Exposé I, Prop. 4.7.3] for equivalence 1. The statement for connections is a direct consequence of this and the fact that a connection is entirely determined by the induced map on global sections. 
the Rees-module associated with $V$. Since it is an $A$-submodule of $V \otimes B$, it is always torsion free. Define the (algebraic) Rees-sheaf $\xi_{\mathbb{A}^{n}}(V)$ to be the coherent sheaf associated to this module, with $\mathbb{G}_{m}^{n}$-equivariant structure corresponding to the grading. This construction is functorial.

It turns out that every toric vector bundle is of the form $\xi_{\mathbb{A}^{n}}(V)$. To formulate this more precisely, we need two definitions:

Definition 7 A splitting for a set of filtrations $F_{1}, \ldots, F_{n}$ on a vector space $V$ is a decomposition

$$
V=\bigoplus_{p \in \mathbb{Z}^{n}} V^{p} \text { s.t. } F_{i}^{r} V=\bigoplus_{\substack{p \in \mathbb{Z}^{n} \\ p_{i} \geq r}} V^{p} .
$$

For $n=1,2$, one can always construct a splitting by choosing appropriate bases. For $n=1$, one sees this by taking successive vector space complements for the inclusions $F^{p+1} \subseteq F^{p}$. For $n=2$, a (notationally more awkward) variant of this method of 'taking complements' still works, but three or more filtrations may or may not be splittable. In fact, given three distinct lines $\ell_{1}, \ldots, \ell_{3} \subseteq k^{2}$, define $F_{i}^{0}:=k^{2}, F_{i}^{1}:=\ell_{i}, F_{i}^{2}:=\{0\}$. Then any splitting would have to involve all three lines as summands, but their sum is not direct. ${ }^{2}$

Definition 8 A map

$$
f:\left(V, F_{1}, \ldots, F_{n}\right) \longrightarrow\left(W, G_{1}, \ldots, G_{n}\right)
$$

in $\mathrm{Fil}_{k}^{n}$ is called $r$-strict if for every collection $\left\{i_{1}, \ldots, i_{r}\right\}$ of indices in $\{1, \ldots, n\}$ and all $r$-tuples of integers $\left(p_{1}, \ldots, p_{r}\right) \in \mathbb{Z}^{r}$,

$$
f\left(F_{i_{1}}^{p_{1}} \cap \cdots \cap F_{i_{r}}^{p_{r}}\right)=G_{i_{1}}^{p_{r}} \cap \cdots \cap G_{i_{r}}^{p_{r}} \cap \operatorname{im}(f) .
$$

E.g., 1-strictness coincides with usual strictness for all filtrations and if there exist splittings for the $F_{i}$ and $G_{i}$ respected by $f$, then $f$ is $n$-strict.

Denoting by $\mathrm{Fil}_{k}^{n, \text { splittable }}$ the full subcategory of $\mathrm{Fil}_{k}^{n}$ consisting of those multifiltered vector spaces that admit a splitting, one has:

Theorem 9 The functor $\xi_{\mathbb{A}^{n}}$ induces an equivalence of categories

$$
\operatorname{Bun}\left(\mathbb{A}^{n}, \mathbb{G}_{m}^{n}\right) \longleftrightarrow \operatorname{Fil}_{k}^{n, \text { splittable }},
$$

which is compatible with direct sums and tensor products and where maps with constant rank on the left side correspond to maps that are $n$-strict on the right hand side. For $n=1,2$ one has $\mathrm{Fil}_{k}^{n}=\mathrm{Fil}_{k}^{n, \text { splittable. }}$.

We will not reproduce a proof here (see e.g. [14, Thm 2.2.1], [13, Thm 1.2.2]), but just explain why the existence of splittings is the right condition on $\xi_{\mathbb{A}^{n}}(V)$ to be a vector bundle and why $n$-strictness corresponds to morphisms of constant rank.

Lemma 10 The functors $\xi_{\mathbb{A}^{n}}\left({ }_{(}\right)$behave as follows when restricted to torus-invariant subsets:

2 c.f. [16, p. 13]. 
1. There is a functorial, $\mathbb{G}_{m}^{n}$-equivariant isomorphism

$$
\left.\xi_{\mathbb{A}^{n}}\left(V, F_{1}, \ldots, F_{n}\right)\right|_{\mathbb{G}_{m} \times \mathbb{A}^{n-1}} \cong \operatorname{pr}_{\mathbb{A}^{n-1}}^{*} \xi_{\mathbb{A}^{n-1}}\left(V, F_{2}, \ldots, F_{n}\right)
$$

and similarly for other subvarieties of the form $\mathbb{A}^{r} \times \mathbb{G}_{m}^{s} \times \mathbb{A}^{t}$. In particular, there is an equivariant isomorphism $\left.\xi_{\mathbb{A}^{n}}(V)\right|_{\mathbb{G}_{m}^{n}} \cong \mathcal{O}_{\mathbb{G}_{m}^{n}} \otimes_{k} V$.

2. Given $\left(V, F_{1}, \ldots, F_{n}\right)$ in $\mathrm{Fil}_{k}^{n}$, set $D^{p}:=\frac{F^{p}}{\sum_{i=1}^{n} F^{p+{ }_{i} 1}}$. There is a functorial isomorphism, equivariant for the $\mathbb{G}_{m}^{n}$-action given by the restricted action on the left and by the grading on the right.

$$
\left.\xi_{\mathbb{A}^{n}}(V)\right|_{\{0\}} \cong \bigoplus_{p \in \mathbb{Z}^{n}} D^{-p}
$$

Proof (Sketch of proof) By Proposition 6, it suffices to give the isomorphisms on the modules of global sections, where restriction to an affine open subset corresponds to a localisation and restriction to a closed subset to a quotient.

Note that, as a vector space, $\operatorname{Rs}^{n}(V)=\bigoplus_{p \in \mathbb{Z}^{n}} F^{p} z^{-p}$. So, if we consider the ideal $I=\left(z_{1}, \ldots, z_{n}\right)$ corresponding to $0 \in \mathbb{A}^{n}$, then the isomorphism in 2 . is given as

$$
\operatorname{Rs}^{n}(V) / I \operatorname{Rs}^{n}(V) \cong \bigoplus_{p \in \mathbb{Z}^{n}} D^{p} z^{-p}
$$

by projection in each summand. Since the grading is given by the $z^{-p}$, we have the sign switch in the statement. As for 1, the restriction corresponds to inverting $z_{1}$. Thus, one has

$$
\begin{aligned}
\operatorname{Rs}^{n}(V)_{z_{1}} & =\bigoplus_{p \in \mathbb{Z}^{n}}\left(\sum_{r \leq p_{1}} F^{\left(r, p_{2}, \ldots, p_{n}\right)}\right) z^{-p} \\
& \cong \bigoplus_{p_{2}, \ldots, p_{n} \in \mathbb{Z}} F^{\left(p_{2}, \ldots, p_{n}\right)} z_{2}^{-p_{2}} \cdot \ldots \cdot z_{n}^{-p_{n}} \otimes_{k} k\left[z_{1}^{ \pm 1}\right] \\
& \cong \operatorname{Rs}^{n-1}\left(V, F_{2}, \ldots, F_{n}\right) \otimes_{k\left[z_{2}, \ldots, z_{n}\right]} k\left[z_{1}^{ \pm}, z_{2}, \ldots, z_{n}\right]
\end{aligned}
$$

where the equality $\sum_{r \leq p_{1}} F^{\left(r, p_{2}, \ldots, p_{n}\right)}=F^{\left(p_{2}, \ldots, p_{n}\right)}$ is a consequence of the assumption that the filtrations are exhaustive (i.e. $F_{1}^{r}=V$ for $r$ small enough).

How to see whether $\xi_{\mathbb{A}^{n}}(V)$ is a vector bundle? For example this is the case if $V=k$ so that each filtration has a single jumping index $r_{i}$, i.e. $F_{i}^{r_{i}+1}=0 \subseteq F_{i}^{r_{i}}=k$. Denoting $r=\left(r_{1}, \ldots, r_{n}\right)$, the Rees module is then just $z^{-r} k\left[z_{1}, \ldots, z_{n}\right]$, which is a free module of rank one. Let us denote the resulting Rees-bundle by $\mathcal{O}_{\mathbb{A}^{n}}(r)$.

Proposition 11 Let $\left(V, F_{1}, \ldots, F_{n}\right)$ be in $\mathrm{Fil}_{k}^{n}$. The following assertions are equivalent:

1. The filtrations $F_{1}, \ldots, F_{n}$ are splittable.

2. $\xi_{\mathbb{A}^{n}}\left(V, F_{1}, \ldots, F_{n}\right)$ is a vector bundle.

3. The spaces $D^{p}=\frac{F^{p}}{\sum_{i=1}^{n} F^{p+{ }_{i} 1}}$ satisfy $\sum_{p \in \mathbb{Z}^{n}} \operatorname{dim} D^{p}=\operatorname{dim} V$.

If one of these conditions is satisfied, any splitting $V=\bigoplus_{p \in \mathbb{Z}^{n}} V^{p}$ determines an isomorphism

$$
\xi_{\mathbb{A}^{n}}(V) \cong \bigoplus_{p \in \mathbb{Z}^{n}} V^{p} \otimes_{k} \mathcal{O}_{\mathbb{A}^{n}}(p)
$$


Proof We first show 1. $\Rightarrow 2 .:$ If $V=\bigoplus V^{p}$ is a splitting of the $F_{i}$, there is an isomorphism $\xi_{\mathbb{A}^{n}}(V)=\bigoplus \xi_{\mathbb{A}^{n}}\left(V^{p}\right) \cong \bigoplus V^{p} \otimes \mathcal{O}_{\mathbb{A}^{n}}(p)$, which is free.

From 2. to 3., note that a vector bundle has constant fibre dimension. By Lemma 10, the fibre over any point (e.g. $(1, \ldots, 1))$ in $\mathbb{G}_{m}^{n}$ is canonically identified with $V$, while the fibre over 0 is canonically identified with $\bigoplus D^{p}$.

Finally, assuming 3., choose, for every $p \in \mathbb{Z}^{n}$, subspaces $V^{p} \subseteq F^{p}$ s.t. $V^{p}$ projects isomorphically onto $D^{p}$. By construction, we have

$$
F^{p}=\sum_{\substack{r \in \mathbb{Z}^{n} \\ r \geq p}} V^{r}
$$

In particular,

$$
\operatorname{dim} V \leq \sum_{p \in \mathbb{Z}^{n}} \operatorname{dim} V^{p}=\sum_{p \in \mathbb{Z}^{n}} \operatorname{dim} D^{p}=\operatorname{dim} V .
$$

Therefore, there has to be an equality and the sum of the $V^{p}$ is direct.

Proposition 12 Let $f: V \longrightarrow W$ be a map in $\mathrm{Fil}_{k}^{n}$ and $\xi_{\mathbb{A}^{n}}(f)$ the associated map of Rees-sheaves.

- The morphism induced by the inclusion $\operatorname{ker} f \hookrightarrow \underline{V}$ gives a canonical identification

$$
\xi_{\mathbb{A}^{n}}(\operatorname{ker} f) \cong \operatorname{ker} \xi_{\mathbb{A}^{n}}(f) .
$$

- There is an exact sequence

$$
0 \longrightarrow T \longrightarrow \operatorname{coker} \xi_{\mathbb{A}^{n}}(f) \stackrel{\varphi}{\longrightarrow} \xi_{\mathbb{A}^{n}}(\operatorname{coker} f) \longrightarrow 0,
$$

where coker $\xi_{\mathbb{A}^{n}}(f)$ is the sheaf-theoretic cokernel and $T$ is the torsion subsheaf. Further,

$$
f \text { is } r \text {-strict } \Leftrightarrow \operatorname{codim}(\operatorname{supp}(T))>r \text {. }
$$

Proof It suffices to check this on global sections, i.e. on the Rees-module. For the first point, note that for any $p \in \mathbb{Z}^{n}$, a section $v_{p} \otimes z^{p}$ with $v_{p} \in F_{V}^{p}$ is mapped to zero iff $v_{p} \in \operatorname{ker} f$.

For the second point, let $\pi: W \rightarrow$ coker $f$ denote the projection. The global sections of the two right members of the claimed sequence are then given (where the sum is direct as $k$-vector spaces) by

$$
\Gamma\left(\mathbb{A}^{n}, \operatorname{coker} \xi_{\mathbb{A}^{n}}(f)\right)=\bigoplus_{p \in \mathbb{Z}^{n}} \frac{F_{W}^{p}}{f\left(F_{V}^{p}\right)} z^{-p}
$$

and

$$
\Gamma\left(\mathbb{A}^{n}, \xi_{\mathbb{A}^{n}}(\operatorname{coker} f)\right)=\bigoplus_{p \in \mathbb{Z}^{n}} \pi F_{W}^{p} z^{-p}
$$

Denoting by $\varphi^{p}: \frac{F_{W}^{p}}{f\left(F_{V}^{p}\right)} \longrightarrow \pi F_{W}^{p}$ the natural map, we can define the map $\varphi$ to be induced by the direct sum of the $\varphi^{p}$. Using

$$
\operatorname{ker} \varphi^{p}=\left\{w \in F_{W}^{p} \mid \exists q \in \mathbb{N}^{n} \text { s.t. } w \in f\left(F_{W}^{p-q}\right)\right\} \bmod f\left(F_{V}^{p}\right),
$$

one verifies that $\operatorname{ker} \varphi=T$ coincides with the torsion subsheaf.

For simplicity, we verify the statement on the dimension of the support of $T$ only in the case $r=n$ : In fact, $n$-strictness is equivalent to the condition that all the $\varphi^{p}$ are isomorphisms, which is in turn equivalent to $\varphi$ being an isomorphism. 


\section{Toric GAGA}

In this section, we are interested in the case $k=\mathbb{C}$ and we only treat vector bundles, not more general equivariant sheaves. We consider equivariant connections and weaker (than being algebraic) regularity conditions on transition functions and group actions.

Notations and conventions: Let $\operatorname{Gra}_{\mathbb{C}}^{n}$ denote the category of finite dimensional $n$ graded $\mathbb{C}$-vector spaces. For $G=\left(\mathbb{C}^{\times}\right)^{n}$ or $G=\mathbb{T}^{n}:=\left(S^{1}\right)^{n}$, by $\operatorname{Bun}^{\omega}\left(\mathbb{C}^{n}, G\right)$ with $\omega \in\{$ alg, hol, sm, cont $\}$, we mean algebraic, holomorphic, smooth or continuous $G$ equivariant bundles on $\mathbb{C}^{n}$ when meaningful. Morphisms are not supposed to have constant rank. We identify Bun $\left(\mathbb{A}^{n}, \mathbb{G}_{m}^{n}\right)$ from the previous section with Bun ${ }^{\text {alg }}\left(\mathbb{C}^{n},\left(\mathbb{C}^{\times}\right)^{n}\right)$ (by considering the complex valued points) and we switch freely between geometric vector bundles and locally free sheaves over the corresponding structure sheaf. By $\operatorname{Rep}^{\omega}(G)$, with $\omega$ as above, we denote algebraic, holomorphic, smooth or continuous representations of $G$. Those cases of interest to us are related to each other by the following diagram:

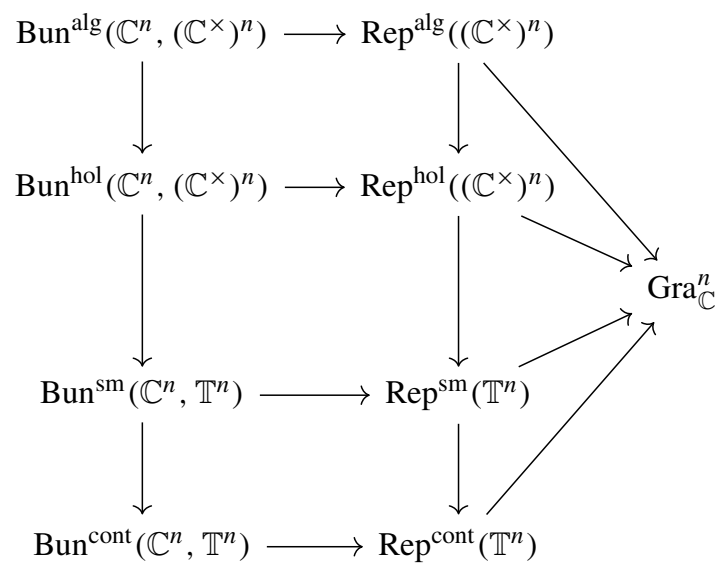

Here, the vertical arrows forget some structure (or in sheaf-theoretic terms, tensor by a bigger structure sheaf and restrict the action), the horizontal ones are restriction to the fibre at 0 and the diagonal ones are given by the rule $(V, \rho) \mapsto V=\bigoplus_{p \in \mathbb{Z}^{n}} V^{p}$ where $V^{p}$ with $p=\left(p_{1}, \ldots, p_{n}\right)$ is the eigenspace of the character

$$
\chi_{p}:\left(\lambda_{1}, \ldots, \lambda_{n}\right) \mapsto \lambda^{-p}:=\lambda_{1}^{-p_{1}} \ldots \lambda_{n}^{-p_{n}} .
$$

Proposition 13 In the diagram (*), all arrows induce bijections on isomorphism classes. The sides of the triangles on the right are equivalences of categories.

Proof It is well-known that all sides of the triangles on the right are equivalences of categories. For the convenience of the reader, let us recall one possible strategy of proof: Let $V$ be some continuous representation of $\mathbb{T}^{n}$. Since $\mathbb{T}^{n}$ is compact, one can choose an invariant scalar product on $V$ and thereby find a decomposition of $V$ into irreducible summands. Since $\mathbb{T}^{n}$ is Abelian, and by Schur's Lemma ([2, p. 69]), stating that maps between two irreducible representations are zero if they are nonisomorphic and a multiple of the identity otherwise, all irreducible components are 1-dimensional. Since $\mathbb{T}^{n}=\left(S^{1}\right)^{n}$, one is thus reduced to classify continuous group homomorphisms $S^{1} \rightarrow S^{1}$ which one checks to be $z \mapsto z^{p}$ for $p \in \mathbb{Z}$. Therefore, $V=\bigoplus_{p \in \mathbb{Z}^{n}} V^{p}$ with $V^{p}$ the eigenspace of $\chi_{p}$. But these are even algebraic and 
defined on $\left(\mathbb{C}^{\times}\right)^{n}$, so the same decomposition holds also in $\operatorname{Rep}^{\omega}(G)$ for $\omega=\operatorname{alg}$, hol, sm. The statement about maps also follows from Schur's Lemma.

To a $G$-representation $(V, \rho)$ in $\operatorname{Rep}^{\omega}(G)$, one can associate the trivial (geometric) vector bundle $\widetilde{V}:=\mathbb{C}^{n} \times V$ with product action

$$
\lambda .(x, v)=(\lambda \cdot x, \rho(\lambda) x) .
$$

We also denote this $\widetilde{V}^{\omega}$ if we want to emphasize that we consider it as a bundle in $\operatorname{Bun}^{\omega}\left(\mathbb{C}^{n}, G\right)$. E.g., in the notation of the previous section, $\widetilde{V}$ alg $=\bigoplus_{p \in \mathbb{Z}^{n}} V^{p} \otimes_{\mathbb{C}} \mathcal{O}_{\mathbb{A}^{n}}$, where as above $V^{p}$ is the eigenspace of $\chi_{p}$, but equipped with the trivial action.

The invariant global sections of $\widetilde{V}^{\omega}$ can be identified with equivariant (algebraic, holomorphic, smooth or continuous) maps $\mathbb{C}^{n} \longrightarrow V$ and the restriction of $\widetilde{V}$ to 0 is canonically isomorphic to $(V, \rho)$, i.e. $V \mapsto \widetilde{V}$ gives a section of the functors from $\operatorname{Bun}^{\omega}\left(\mathbb{C}^{n}, G\right)$ to $\operatorname{Rep}^{\omega}(G)$. In particular, all horizontal arrows in $(*)$ are essentially surjective.

Because $\mathbb{C}^{n}$ is $\mathbb{T}^{n}$-equivariantly contractible to the fixed point 0 , the restriction to the fibre

$$
\operatorname{Bun}^{\omega}\left(\mathbb{C}^{n}, \mathbb{T}^{n}\right) \longrightarrow \operatorname{Rep}^{\omega}\left(\mathbb{T}^{n}\right)
$$

induces a bijection on isomorphism classes ${ }^{3}$ for $\omega \in\{\mathrm{sm}$, cont $\}$ and by Theorem 9 and Proposition 11, so does

$$
\operatorname{Bun}^{\operatorname{alg}}\left(\mathbb{C}^{n},\left(\mathbb{C}^{\times}\right)^{n}\right) \longrightarrow \operatorname{Gra}_{\mathbb{C}}^{n}
$$

Finally, by a general Theorem of Heinzner and Kutzschebauch on equivariant bundles on Stein spaces ${ }^{4}$ the forgetful functor

$$
\operatorname{Bun}^{\text {hol }}\left(\mathbb{C}^{n},\left(\mathbb{C}^{\times}\right)^{n}\right) \longrightarrow \operatorname{Bun}^{\text {cont }}\left(\mathbb{C}^{n}, \mathbb{T}^{n}\right)
$$

induces a bijection on isomorphism classes. The remaining arrows are bijections on isomorphism classes by commutativity of the diagram induced by (*) on isomorphism classes.

For the functor from algebraic to holomorphic bundles, even more is true:

Proposition 14 The functor

$$
\operatorname{Bun}^{a l g}\left(\mathbb{C}^{n},\left(\mathbb{C}^{\times}\right)^{n}\right) \longrightarrow \operatorname{Bun}^{h o l}\left(\mathbb{C}^{n},\left(\mathbb{C}^{\times}\right)^{n}\right)
$$

is an equivalence of categories.

Proof We already know that it is essentially surjective by Proposition 13. It is also obviously faithful, so what remains to be checked is that it is full. Consider a map of equivariant holomorphic bundles $\mathcal{V} \longrightarrow \mathcal{W}$. Without loss of generality, we may assume $\mathcal{V}=\widetilde{V}, \mathcal{W}=\widetilde{W}$ for some $\left(\mathbb{C}^{\times}\right)^{n}$-representations $V, W$. In this case, the statement follows from the following lemma applied to the bundle $\operatorname{Hom}(\mathcal{V}, \mathcal{W})$.

\footnotetext{
3 This follows from the 'homotopy invariance of isomorphism classes of equivariant vector bundles': Given a compact topological group $G$ and two equivariantly homotopic equivariant maps $f_{0}, f_{1}: X \longrightarrow Y$ between $G$-spaces with $X$ paracompact. Then for any vector bundle $\mathcal{V}$ on $Y$ the pullbacks $f_{0}^{*} \mathcal{V}$ and $f_{1}^{*} \mathcal{V}$ are equivariantly isomorphic. This is essentially proven in [1], p. 40f. However, there only the case of a compact Hausdorff base and a finite group is treated. See [21] for the case of a compact group (but still compact base) and, e.g., [26, p. 21] for the case of non-equivariant bundles over a paracompact base, which can be adapted to the equivariant case by a standard averaging trick.

${ }^{4}$ [9, p. 341], see also [8, par. 1.4.] for the notions used in the statement.
} 
Lemma 15 Let $V$ be an object of $\operatorname{Rep}^{\text {hol }}\left(\left(\mathbb{C}^{\times}\right)^{n}\right)$. For any equivariant section $z \mapsto(z, s(z))$ of the bundle $\widetilde{V}^{\text {hol }}$, there are a finite subset $I \subseteq \mathbb{Z}_{\geq 0}^{n}$ and elements $v_{p} \in V^{-p}$ for all $p \in I$ s.t.

$$
s=\sum_{p \in I} z^{p} v_{p}
$$

Proof It suffices to check the equality on the dense open subset $U=\left(\mathbb{C}^{\times}\right)^{n} \subseteq \mathbb{C}^{n}$. Since $\left(\mathbb{C}^{\times}\right)^{n}$ acts simply transitively on $U$, a section is determined by its value $s(1, \ldots, 1)=$ $\sum_{p \in I} v_{p}$. Because the section extends to the whole of $\mathbb{C}^{n}$, necessarily $I \subseteq \mathbb{Z}_{\geq 0}^{n}$. This implies the lemma.

Remark 16 The other two forgetful functors are still faithful and essentially surjective, but no longer full: E.g. consider any equivariant line bundle $L$. Then $\operatorname{Hom}(L, L)$ is the (equivariantly) trivial bundle. An $S^{1}$-invariant continuous section $s$ of the trivial bundle $\mathbb{C} \times \mathbb{C}$ can be given by prescribing an arbitrary continuous function $\mathbb{R}_{\geq 0} \rightarrow \mathbb{C}$ (the restriction to $\left.s\right|_{\mathbb{R}_{\geq 0}}$ ), but these sections do not have to be smooth, and even if they are, they don't have to be constant (i.e. $\mathbb{C}^{\times}$-equivariant).

Remark 17 Using the whole diagram (*) and in particular [9] to show essential surjectivity of analytification seems to be quite an overkill. It would be interesting to see a direct derivation of the classification of isomorphism classes in $\operatorname{Bun}^{\text {hol }}\left(\mathbb{C}^{n},\left(\mathbb{C}^{\times}\right)^{n}\right)$.

Theorem 18 For any smooth toric variety $X$, analytification yields an equivalence of categories

$$
\left\{\begin{array}{l}
\text { equivariant algebraic vector } \\
\text { bundles on } X
\end{array}\right\} \longleftrightarrow\left\{\begin{array}{l}
\text { equivariant holomorphic } \\
\text { vector bundles on } X^{\text {an }}
\end{array}\right\}
$$

Proof For any groups $G, G^{\prime}$ and $G$-space $X$, one obtains by pullback an identification of the $G \times G^{\prime}$-equivariant bundles over $X \times G^{\prime}$ and the $G$-equivariant bundles over $X$. In fact, any equivariant bundle on $X \times G^{\prime}$ is trivialized in the fibre direction by the action of $G^{\prime}$, so one can pick transition functions depending only on the base. Also, an invariant section has to be constant along the fibre. In particular, equivariant maps (invariant sections of a homomorphism bundle) of bundles on $X \times G^{\prime}$ correspond to equivariant maps between bundles on $X$. This reasoning works regardless whether one works in the algebraic or holomorphic category. In particular, there is an equivalence

$$
\operatorname{Bun}^{\omega}\left(\mathbb{C}^{n} \times\left(\mathbb{C}^{\times}\right)^{m},\left(\mathbb{C}^{\times}\right)^{n+m}\right) \longrightarrow \operatorname{Bun}^{\omega}\left(\mathbb{C}^{n},\left(\mathbb{C}^{\times}\right)^{n}\right)
$$

for $\omega=\mathrm{alg}$, hol and so by Proposition 14, analytification gives an equivalence of algebraic and holomorphic equivariant bundles over $\mathbb{C}^{n} \times\left(\mathbb{C}^{\times}\right)^{m}$. One concludes by noting that every toric variety is covered by open sets equivariantly isomorphic to $\mathbb{C}^{n} \times\left(\mathbb{C}^{\times}\right)^{m}$ for some $n, m \in \mathbb{Z}_{\geq 0}$, the intersections of which have again this form. ${ }^{5}$

Corollary 19 Let X be a smooth toric variety. Analytification yields an equivalence of categories

$$
\left\{\begin{array}{l}
\text { equivariant algebraic vector } \\
\text { bundles with an equivariant connection on } X
\end{array}\right\} \longleftrightarrow\left\{\begin{array}{l}
\text { equivariant holomorphic } \\
\text { vector bundles with an } \\
\text { equivariant connection on } X^{\text {an }}
\end{array}\right\} .
$$

It restricts to an equivalence of the subcategories of vector bundles with flat connections.

$\overline{5}$ See [3, thm. 3.1.19, thm. 1.3.12, ex. 1.2.21]. 
Proof That the functor is fully faithful follows from the corresponding statement for bundles without a connection. In fact, a map between algebraic vector bundles with connection is compatible with the connections if and only if its analytification is. Similarly, a connection is flat if and only if its analytification is. For essential surjectivity, we can assume that $X=\mathbb{C}^{n} \times\left(\mathbb{C}^{\times}\right)^{m}$ with action by multiplication of $\left(\mathbb{C}^{\times}\right)^{n+m}$ and that a holomorphic bundle $\mathcal{V}$ is given in trivialized form, i.e., as $\mathcal{V}=\operatorname{pr}_{\mathbb{C}^{n}}^{*} \widetilde{\mathcal{V}(0)} \otimes \operatorname{pr}_{\left(\mathbb{C}^{\times}\right)^{m}}^{*} \mathcal{O}_{\left(\mathbb{C}^{\times}\right)^{m}}$. One checks that on these (trivial) bundles the canonical connection $d$ is equivariant, so any connection is given as

$$
\nabla=d+\Omega,
$$

where $\Omega$ is a global invariant holomorphic 1 -form on $\mathbb{C}^{n} \times\left(\mathbb{C}^{\times}\right)^{m}$ with values in the vector space $\operatorname{End}(\mathcal{V}(0))$. Arguing as in the proof of Lemma 15, such a form can be written as

$$
\Omega=\sum_{p \in \mathbb{Z}_{\geq 0}^{n}} \sum_{i=1}^{n+m} A_{p, i} z_{1}^{p_{1}} \cdot \ldots \cdot z_{n}^{p_{n}} \frac{d z_{i}}{z_{i}},
$$

where the $A_{p, i}$ are endomorphisms of $\mathcal{V}(0)$ of multidegree $-p$ (which are taken to be zero if they would cause a pole, i.e., if $p_{i}=0$ ). In particular, the connection is algebraic.

For flat connections, there is also a nicer comparison to representations, which also accounts for the smooth case. Let $\operatorname{Bun}_{\nabla}^{\omega}\left(\mathbb{C}^{n},\left(\mathbb{C}^{\times}\right)^{n}\right)$ with $\omega \in\{\mathrm{alg}$, hol $\}$ denote the category of $\left(\mathbb{C}^{\times}\right)^{n}$ algebraic or holomorphic equivariant vector bundles with an equivariant connection and denote by $\operatorname{Bun}_{\nabla b}^{\omega}\left(\mathbb{C}^{n},\left(\mathbb{C}^{\times}\right)^{n}\right)$ the respective subcategories of flat connections. Let $\operatorname{Bun}_{\nabla^{b}}^{\text {sm }}\left(\mathbb{C}^{n}, \mathbb{T}^{n}\right)$ be the category of smooth equivariant vector bundles with flat equivariant connections. As above, there is a commutative diagram:

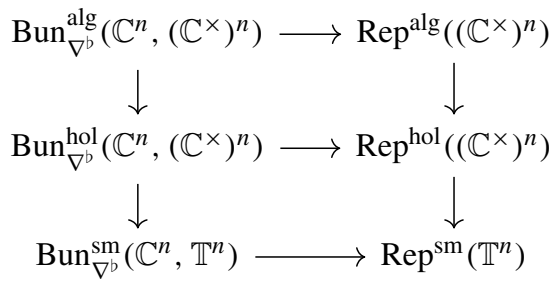

Here, horizontal arrows are again restriction to 0 and vertical ones forget about the stronger regularity conditions imposed.

Proposition 20 In the diagram (**), all arrows are equivalences of categories.

Proof Since we already know the functors in the right column and the one from algebraic to holomorphic bundles to be an equivalence, it suffices to show that restriction to the fixed point is an equivalence in the holomorphic and smooth cases. We do this in the holomorphic case, the smooth case works the same way.

Sending $(V, \nabla)$ to ker $\nabla$ is an equivalence of categories between flat equivariant connections and equivariant local systems on $\mathbb{C}^{n}$. Since $\mathbb{C}^{n}$ is contractible, any local system is necessarily trivial. So restriction from global sections to any point is an isomorphism. In particular, restriction to the fixed point 0 induces an equivalence of categories with $\operatorname{Rep}^{\text {hol }}\left(\left(\mathbb{C}^{\times}\right)^{n}\right)$. 
For completeness, here is an explicit description of the pseudo-inverse to the restriction functor in $(* *)$ : For any representation $(V, \rho)$ in $\operatorname{Rep}^{\text {hol }}\left(\left(\mathbb{C}^{\times}\right)^{n}\right)$, as before, consider the bundle $\widetilde{V}$ with product action. Its sheaf of sections $V \otimes_{\mathbb{C}} \mathcal{O}_{\mathbb{C}^{n}}$ is equipped with the canonical equivariant connection $d$ given by

$$
d(v \otimes f)=v \otimes d f
$$

and sending a map $f:\left(V, \rho_{V}\right) \longrightarrow\left(W, \rho_{W}\right)$ of representations to $f \otimes$ Id this defines the pseudo-inverse

$$
\operatorname{Rep}^{\text {hol }}\left(\left(\mathbb{C}^{\times}\right)^{n}\right) \longrightarrow \operatorname{Bun}_{\nabla^{b}}\left(\mathbb{C}^{n},\left(\mathbb{C}^{\times}\right)^{n}\right)
$$

Remark 21 It is a natural task to extend these results to arbitrary coherent sheaves and possibly singular toric varieties. The coherent sheaf case might be approachable by the same methods as in this article: One would want some sort of equivariant resolution by vector bundles in the holomorphic setting (whose existence the author is currently unaware of). For the case of singular base, the present method of reducing to $\mathbb{C}^{n}$ appears to be unsuitable.

Remark 22 An early impetus for the questions treated in this section came from studying Kapranov's proof [12] of the equivalence between (complex) Mixed Hodge Structures and algebraic toric vector bundles with an equivariant connection on $\mathbb{C}^{2}$. A crucial step in the proof consists in an application of the equivariant Radon-Penrose transform, that yields an equivalence between equivariant holomorphic vector bundles with a connection on $\mathbb{C}^{2}$ and a certain holomorphic toric vector bundle on $\mathbb{P}_{\mathbb{C}}^{2} \backslash\{[1,0,0]\}$. The latter are then related to triples of opposed filtrations via the Rees-bundle construction. One then has to check that if a bundle on one side of the equivalence is algebraic, so is its counterpart on the other side. By Theorem 18 and Corollary 19 this last step is in a certain sense redundant: The algebraic and holomorphic categories on both sides are equivalent.

\section{The Frölicher approximating bundle}

For a complex manifold $X$, denote by $\left(C^{\infty}(X, \mathbb{C}), d\right)$ the complex of $\mathbb{C}$-vector spaces given by complex-valued differential forms and exterior differentiation. This complex carries a filtration given by

$$
F^{p} C^{\infty}(X, \mathbb{C}):=\bigoplus_{\substack{r+s=. \\ r \geq p}} C_{p, q}^{\infty}(X, \mathbb{C}),
$$

where $C_{p, q}^{\infty}(X, \mathbb{C})$ denotes the subspace of forms of type $p, q$. This filtration induces a filtration on $H_{d R}^{k}(X, \mathbb{C})$, still denoted $F^{\bullet}$, by

$$
F^{p} H_{d R}^{k}(X, \mathbb{C}):=\operatorname{im}\left(\operatorname{ker} d \cap F^{p} C_{k}^{\infty}(X, \mathbb{C}) \longrightarrow H_{d R}^{k}(X, \mathbb{C})\right) .
$$

The filtration $F$ on $C_{\bullet}^{\infty}(X, \mathbb{C})$ induces the so-called Frölicher spectral sequence

$$
E_{1}^{p, q}(X)=H^{q}\left(X, \Omega_{X}^{p}\right) \Longrightarrow\left(H_{d R}^{p+q}(X, \mathbb{C}), F\right) .
$$


In [19] (from which we adopt some notations in this section), Dan Popovici associates with every compact connected complex manifold $X$ a holomorphic vector bundle, called the Frölicher approximating vector bundle (short FAVB), denoted $\mathcal{A}^{k}$ on $\mathbb{C}$ together with distinguished isomorphisms of the fibres:

$$
\psi_{h}: \mathcal{A}_{h}^{k} \cong \begin{cases}H_{d R}^{k}(X, \mathbb{C}) & \text { if } h \neq 0 \\ \bigoplus_{p+q=k} E_{\infty}^{p, q}(X) & \text { if } h=0 .\end{cases}
$$

Here, $E_{\infty}(X)$ denotes the limiting page of the Frölicher spectral sequence of $X$. Recall that convergence of the spectral sequence means that the separated and exhaustive descending filtration $F$ on $H_{d R}^{k}(X, \mathbb{C})$ satisfies $E_{\infty}^{p, q}=\operatorname{gr}_{F}^{p} H_{d R}^{p+q}$. Further, $E_{\infty}^{p, q} \cong E_{r}^{p, q}$ for any $r$ with the property that all differentials entering or leaving the bidegree $(p, q)$ are zero from page $E_{r}$ onward. In particular, one could replace $\infty$ in the statement with the minimal $r_{0}$ s.t. the spectral sequence degenerates at $E_{r_{0}}$ (e.g. one always has $\left.r_{0} \leq \operatorname{dim}_{\mathbb{C}} X+1\right)$.

Popovici's construction depends on the choice of a metric on $X$. Let us briefly recall it: The main point consists in contructing a $C^{\infty}$-family of Laplace-type pseudo-differential operators $\left(\tilde{\Delta}_{h}\right)_{h \in \mathbb{C}}$ on the space of $k$-forms $C_{k}^{\infty}(X, \mathbb{C})$. The kernels of $\tilde{\Delta}_{h}$ form a $C^{\infty}$-vector bundle over $\mathbb{C}$. Then one computes that inclusion and projection induce isomorphisms

$$
\text { ker } \tilde{\Delta}_{h} \cong \begin{cases}H_{d_{h}}^{k}(X, \mathbb{C}) & \text { if } h \neq 0 \\ \bigoplus_{p+q=k} E_{\infty}^{p, q}(X) & \text { if } h=0 .\end{cases}
$$

Here, $H_{d_{h}}(X, \mathbb{C})$ denotes cohomology with respect to the 'twisted' differential $d_{h}=h \partial+\bar{\partial}$. For every $0 \neq h \in \mathbb{C}$, the map

$$
\begin{aligned}
\theta_{h}: C_{k}^{\infty}(X, \mathbb{C}) & \longrightarrow C_{k}^{\infty}(X, \mathbb{C}) \\
\sum_{p+q=k} \omega^{p, q} & \longmapsto \sum_{p+q=k} h^{p} \omega^{p, q}
\end{aligned}
$$

induces isomorphisms $H_{d R}^{k}(X, \mathbb{C}) \cong H_{d_{h}}^{k}(X, \mathbb{C})$. This varies holomorphically in $h$, hence the bundle ker $\tilde{\Delta}$ is holomorphic over $\mathbb{C}^{\times}$(even a constant local system). Therefore, it is necessarily also holomorphic over the whole of $\mathbb{C}$ by Riemann's removable singularity theorem.

We show now that $\mathcal{A}^{k}$ can be identified with a Rees-bundle. Slightly abusing notation, we identify $\mathcal{A}^{k}$ with its sheaf of sections.

Theorem 23 Let $X$ be a compact connected complex manifold and $\mathcal{A}^{k}$ the $k$-th Frölicher approximating bundle. There is a canonical isomorphism

$$
\mathcal{A}^{k} \cong \xi_{\mathbb{A}^{1}}\left(H_{d R}^{k}(X, \mathbb{C}), F\right)^{\text {an }}
$$

where $F$ denotes the Hodge filtration and the superscript ${ }^{\text {an }}$ means analytification.

Corollary 24 The bundle $\mathcal{A}^{k}$ carries a (necessarily unique) algebraic and $\mathbb{C}^{\times}$-equivariant structure which is independent of the choice of a metric. The association $X \longmapsto \mathcal{A}^{k}$ is contravariantly functorial for maps between (compact) complex manifolds.

Note that the Rees-bundle construction makes perfect sense for infinite dimensional vector spaces, yielding a quasi-coherent (and for one filtration or two filtrations with the same proof 
as in Proposition 11: locally free) sheaf. Hence, one can also omit the compactness condition and use $\xi_{\mathbb{A}^{1}}\left(H_{d R}^{k}(X, \mathbb{C}), F\right)$ as a definition of the FAVB for arbitrary complex manifolds (although one may argue that the filtration used here is not the 'right' filtration to consider in the non-compact case).

By Lemma 10 , the bundle $\xi_{\mathbb{A}^{1}}\left(H_{d R}^{k}(X, \mathbb{C}), F\right)$ has the desired identifications $(*)$. The isomorphism with $\mathcal{A}^{k}$ will follow from the following general 'base-change-property' for Rees-bundles.

Lemma 25 Given a complex manifold $X$ and $k \in \mathbb{Z}$, consider the complex $\left(A_{\bullet}, d_{\xi}\right)$ of sheaves on $\mathbb{C}$ defined by $A .=\xi_{\mathbb{A}^{1}}\left(C_{\bullet}^{\infty}(X, \mathbb{C}), F\right)$ and $d_{\xi}=\xi_{\mathbb{A}^{1}}(d)$. There is a canonical identification of toric vector bundles on $\mathbb{C}$ :

$$
H^{k}\left(A_{\bullet}^{\mathrm{an}}, d_{\xi}^{\mathrm{an}}\right) / T \cong \xi_{\mathbb{A}^{1}}\left(H_{d R}^{k}(X, \mathbb{C}), F\right)^{\mathrm{an}}
$$

where $T$ denotes the torsion subsheaf.

There are canonical isomorphisms

$$
\begin{aligned}
\alpha_{k}: A_{k} & \longrightarrow C_{k}^{\infty}(\widetilde{X, \mathbb{C}) \otimes \mathbb{C}[z]} \\
\sum_{p+q=k} \omega^{p, q} \cdot p\left(z^{ \pm 1}\right) & \longmapsto \sum_{p+q=k} \omega^{p, q} p\left(z^{ \pm 1}\right) z^{p}
\end{aligned}
$$

under which $d_{\xi}$ gets identified with $d_{z}=z \cdot(\partial \otimes \mathrm{Id})+(\bar{\partial} \otimes \mathrm{Id})$.

Proof The first part is a direct application of Proposition 12, i.e. the fact that the Reesconstruction commutes with kernels and commutes with cokernels up to torsion. The isomorphism in the second part is the trivialization from Proposition 11 (note that there is the canonical splitting of $F$ given by $C_{k}^{\infty}(X, \mathbb{C})=\bigoplus_{p+q=k} C_{p+q}^{\infty}(X, \mathbb{C})$ ).

Proof of Theorem 23 Consider the holomorphic bundle ker $\tilde{\Delta}_{z}$ as a subsheaf of $C_{k}^{\infty}(X, \mathbb{C}) \otimes_{\mathbb{C}}$ $\mathcal{O}_{\mathbb{C}}$. By equation (**), it is contained in $\operatorname{ker} d_{z} \cap C_{k}^{\infty}(X, \mathbb{C}) \otimes_{\mathbb{C}} \mathcal{O}_{\mathbb{C}} \cong A_{k} \cap \operatorname{ker} d_{\xi}$ and therefore projects to $H^{k}\left(A_{\bullet}^{\mathrm{an}}, d_{\xi}^{\text {an }}\right) \bmod T$. The result now follows from $(*)$ and Lemma 10.

We end this section and the article by sketching a few open ends and questions:

- (Second filtration) The FAVB bundle does not see the real structure on $H_{d R}^{k}(X, \mathbb{C})$. To remedy this, one can take the conjugate Hodge-filtration into account and obtain a bundle on $\mathbb{A}^{2}$, given as $\xi_{\mathbb{A}^{2}}\left(H_{d R}^{k}(X, \mathbb{C}), F, \bar{F}\right)$, which is now also equivariant with respect $\mathbb{G}_{m}^{2}$ and the antilinear involution on the base given by $\left(z_{1}, z_{2}\right) \mapsto\left(\bar{z}_{2}, \bar{z}_{1}\right)$ and hence descends to a real bundle on $\mathbb{A}_{\mathbb{R}}^{2}$. One can also form $\xi_{\mathbb{P}^{1}}\left(H_{d R}^{k}(X, \mathbb{C}), F, \bar{F}\right)$, i.e. descend the bundle to projective space. If the Hodge-filtrations induce a pure Hodge structure of some weight $k^{\prime}$ (not necessarily $=k$ ) on $k$, this is a Twistor Structure in the sense of Simpson [24] (after forgetting most of the action). Let us instead continue to consider it as a bundle on $\mathbb{C}^{2}$. Its restriction to $\mathbb{A}^{1} \times\{h\}(h \neq 0)$ yields the FAVB, while its restriction to $\{h\} \times \mathbb{A}^{1}$ $(h \neq 0)$ yields the analogous bundle for the conjugate spectral sequence. It is, similarly to Proposition 25, isomorphic to the cohomology (modulo torsion) of the de-Rham complex with parameters $z_{1}, z_{2}$ and deformed differential, now given by $z_{1} \partial+z_{2} \bar{\partial}$. The fibre of $\xi_{\mathbb{A}^{2}}\left(H_{d R}^{k}(X, \mathbb{C}), F, \bar{F}\right)$ over $(0,0)$ is given by the space

$$
D=\bigoplus_{p, q \in \mathbb{Z}} \frac{F^{p} \cap \bar{F}^{q}}{F^{p+1} \cap \bar{F}^{q}+F^{p} \cap \bar{F}^{q+1}}
$$


The dimensions $b_{k}^{p, q}$ of the bigraded components of $D$ are called the generalized Betti numbers and satisfy $b_{k}(X)=\sum_{p, q} b_{k}^{p, q}(X)$. For $p+q \neq k$, their nonvanishing is an obstruction to the de-Rham cohomology being pure (meaning the existence of a decomposition $\left.H_{d R}^{k}(X, \mathbb{C})=\bigoplus_{p+q=k} F^{p} \cap \bar{F}^{q}\right)$, see also [25] for a more conceptual interpretation. Can one also describe this bundle, arising from two filtrations, as the kernel of a family of differential operators? In particular, is there a harmonic theory for (the bigraded components of) the space $D$ ? These questions might be more approachable for the class of page- $r-\partial \bar{\partial}$-manifolds introduced in [20].

- (Relative version) In [19] Popovici also considers the situation of a family of compact complex manifolds i.e. a proper holomorphic submersion $\pi: X \longrightarrow B$ and constructs a bundle $\mathcal{A}^{k}$ on $\mathbb{C} \times B$, s.t. the restriction to each slice $\mathbb{C} \times\{b\}$ is the FAVB for $X_{b}=\pi^{-1}(b)$. Is there also a purely algebraic construction for this bundle? To make the Rees-bundle construction work in the relative setting, one maybe should answer the following question: Are the $F^{p} H_{d R}^{k}\left(X_{b}, \mathbb{C}\right)$ fibres of a coherent subsheaf of the (flat) holomorphic vector bundle $R^{k} \pi_{*} \mathbb{C} \otimes \mathcal{O}_{B}$ ? It would also be interesting to consider the two-filtration version above in the relative setting.

Acknowledgements This article was written at LMU München. Part of the material is also contained in my PhD-thesis, elaborated at WWU Münster with financial support by the SFB 878. I warmly thank Christopher Deninger, Jörg Schürmann and Dan Popovici for interesting and encouraging discussions and advice. Finally, I am grateful to the anonymous referee for the very careful reading and many good comments and suggestions.

Funding Open Access funding enabled and organized by Projekt DEAL.

Open Access This article is licensed under a Creative Commons Attribution 4.0 International License, which permits use, sharing, adaptation, distribution and reproduction in any medium or format, as long as you give appropriate credit to the original author(s) and the source, provide a link to the Creative Commons licence, and indicate if changes were made. The images or other third party material in this article are included in the article's Creative Commons licence, unless indicated otherwise in a credit line to the material. If material is not included in the article's Creative Commons licence and your intended use is not permitted by statutory regulation or exceeds the permitted use, you will need to obtain permission directly from the copyright holder. To view a copy of this licence, visit http://creativecommons.org/licenses/by/4.0/.

\section{References}

1. Atiyah, M.F.: $K$-Theory. Benjamin Inc, W. A., New York (1967)

2. Bröcker, T., tom Dieck, T.: Representations of Compact Lie Groups. Graduate Texts in Mathematics. Springer, New York (1985)

3. Cox, D.A., Little, J.B., Schenck, H.K.: Toric Varieties. Graduate Studies in Mathematics, vol. 134. American Mathematical Society, Washington, DC (2011)

4. Demazure, M., Grothendieck, A.: (SGA III) Schémas en groupes, Tome I: Propriétés générales des schémas en groupes, Exposés I-VII, (Séminaire de Géométrie Algébrique du Bois Marie 1962-1964). No. 151 in Lecture Notes in Math. Springer (1970)

5. Deninger, C.: On the $\Gamma$-factors attached to motives. Invent. Math. 104(2), 245-262 (1991)

6. Deninger, C.: On the $\Gamma$-factors of motives II. Doc. Math. 6, 69-97 (2001)

7. Goncharov, A.B.: Hodge correlators. (2008). arXiv:0803.0297 [math]

8. Heinzner, P.: Geometric invariant theory on Stein spaces. Math. Ann. 289(4), 631-662 (1991)

9. Heinzner, P., Kutzschebauch, F.: An equivariant version of Grauert's Oka principle. Invent. Math. 119(1), 317-346 (1995)

10. Kaneyama, T.: On equivariant vector bundles on an almost homogeneous variety. Nagoya Math. J. 57, 65-86 (1975)

11. Kaneyama, T.: Torus-equivariant vector bundles on projective spaces. Nagoya Math. J. 111, 25-40 (1988)

12. Kapranov, M.: Real mixed Hodge structures. J. Noncommut. Geom. 6(2), 321-342 (2012)

13. Klyachko, A.: Vector bundles and torsion free sheaves on the projective plane. MPIM Preprint (1991) 
14. Klyachko, A.A.: Equivariant bundles on toral varieties. Math. USSR Izv. 35(2), 337 (1990)

15. Krishna, A., Ravi, C.: Equivariant vector bundles, their derived category and K-theory on affine schemes. Ann. K-Theory 2(2), 235-275 (2016)

16. Penacchio, O.: Structures de Hodge mixtes et fibrés sur le plan projectif complexe. PhD thesis, July (2003)

17. Penacchio, O.: Mixed Hodge structures and equivariant sheaves on the projective plane. Math. Nachr. 284(4), 526-542 (2011)

18. Perling, M.: Graded rings and equivariant sheaves on toric varieties. Math. Nachr. 263(1), 181-197 (2004)

19. Popovici, D.: Adiabatic limit and deformations of complex structures. (2019). arXiv:1901.04087 [math]

20. Popovici, D., Stelzig, J., Ugarte, L.: Higher-page Hodge theory of compact complex manifolds. (2020). arXiv:2001.02313 [math]

21. Segal, G.: Equivariant $k$-theory. Publ. Math. l'IHÉS 34, 129-151 (1968)

22. Serre, J.-P.: Géométrie algébrique et géométrie analytique. Ann. l’Inst. Fourier 6, 1-42 (1956)

23. Simpson, C.: The Hodge filtration on nonabelian cohomology. Algebraic Geom. Santa Cruz 1995 62, 217-281 (1997)

24. Simpson, C.: Mixed twistor structures. (1997). arXiv:alg-geom/9705006

25. Stelzig, J.: On the structure of double complexes. (2018). arXiv:1812.00865 [math]

26. Zois, I.P.: 18 Lectures on K-Theory. (2010). arXiv:1008.1346 [math]

Publisher's Note Springer Nature remains neutral with regard to jurisdictional claims in published maps and institutional affiliations. 